\title{
Metabolomics of Breast Milk: The Importance of Phenotypes
}

\author{
Angelica Dessì ${ }^{1, *, \dagger}$, Despina Briana ${ }^{2, \dagger}$, Sara Corbu ${ }^{1}$, Stavroula Gavrili ${ }^{3}$, \\ Flaminia Cesare Marincola ${ }^{4}{ }^{\mathbb{D}}$, Sofia Georgantzi ${ }^{3}$, Roberta Pintus ${ }^{1}$ (D), Vassilios Fanos ${ }^{1}$ and \\ Ariadne Malamitsi-Puchner ${ }^{2}$ \\ 1 Neonatal Intensive Care Unit, Neonatal Pathology and Neonatal Section, Azienda University Polyclinic, \\ University of Cagliari, 09124 Cagliari, Italy; sari.crb@gmail.com (S.C.); gomberta@icloud.com (R.P.); \\ vafanos@tin.it (V.F.) \\ 2 National and Kapodistrian University of Athens, 10679 Athens, Greece; brianadespina@yahoo.com (D.B.); \\ amalpu@med.uoa.gr (A.M.-P.) \\ 3 Neonatal Intensive Care Unit, General District, Hospital Alexandra, 11528 Athens, Greece; \\ vpgavrili@gmail.com (S.G.); sofiageorgantzi@yahoo.gr (S.G.) \\ 4 Department of Chemical and Geological Sciences, University of Cagliari, 09124 Cagliari, Italy; \\ flaminia@unica.it \\ * Correspondence: angelicadessi@unica.it; Tel.: +39-070-5109-3403 \\ + The authors contributed equally to the work.
}

Received: 25 October 2018; Accepted: 17 November 2018; Published: 20 November 2018

\begin{abstract}
Breast milk is the gold standard of nutrition for newborns. Its composition is tailored to the nutritional needs of the infant and varies between mothers. In recent years, several bioactive molecules have been discovered in addition to the main nutrients, such as multipotent stem cells, hormones, immunoglobulins, and bacteria. Furthermore, the human milk oligosaccharides (HMOs) seem to exert several important protective biological functions. According to the HMOs' composition, breast milk can be classified as a secretory or non-secretory phenotype. In our study, we investigated the metabolome of milk collected from 58 mothers that delivered neonates at term, that were appropriate, small or large for gestational age, by performing nuclear magnetic resonance spectroscopy ( $\left.{ }^{1} \mathrm{H}-\mathrm{NMR}\right)$. From the data analysis, two groups were distinguished based on their different types of oligosaccharides, and classified according the mother phenotype: secretory and non-secretory. This information is of major importance given the different biological function of the different HMOs, such as immune-modulation and protection against disease. This would allow us to predict whether the neonate would be, for instance, more prone to developing certain diseases, and to tailor her or his nutrition to fit their needs perfectly and pave the way to a personalized nutrition.
\end{abstract}

Keywords: metabolomics; human milk; NMR

\section{Introduction}

Maternal milk is a complex fluid that evolutionarily adapted to satisfy the nutritional needs of the neonate. In addition to the classical nutrients, such as proteins, carbohydrates, lipids, vitamins, and minerals, milk contains several bioactive components [1]. Among them there are growth factors, anti-microbial components, and stem cells, which are able to integrate in vivo in the tissues of the neonate and to differentiate in mature cells [2].

Human breast milk (HBM) has the characteristic of significantly varying from one woman to another, and it changes constantly during lactation to adapt to the growing energy needs of the developing infant. The variable components of HBM include oligosaccharides, which represent the 
third most abundant constituent. They exert several important functions, such as the modulation of the composition of the gut microbiota that influences a wide range of physiological processes in the neonate [3]. In fact, oligosaccharides are indigestible by the neonate, but they are a source of nourishment for the bacteria colonising and living in the neonate's guts. Their presence can favor the growth of some species of commensal bacteria (i.e., Bifidobacteria strains) and inhibit the growth of pathogens. Furthermore, Bifidobacteria spp. can also exert direct antibacterial activity by modulating binding receptors, both on the pathogens and on the gut lumen blocking their binding and eventual damage. Bifidobacteria spp. also modify the cytokine response in T-cells. Moreover, since the types of oligosaccharides present in human milk is genetically determined, some profiles are thought to be more protective than others, even against respiratory tract infections. Finally, it is interesting to notice that according to a recent study, oligosaccharides are not altered by pasteurization or freeze-drying [4].

The application of metabolomics in neonatology offers an approach to investigate the complex relationships between nutrition and infant's health. The characterization of the metabolome of HBM compared to formula milk allows understanding of how each nutrient affects the neonate's metabolism, and offers the chance to intervene on the composition of the diet according to the nutritional request of the neonate [5-9].

In this study, we compared the metabolome of HBM samples collected from women that delivered neonates appropriately large or small for their gestational age and weight, in order to explore the presence of possible differences correlated to the fetal growth. The metabolomics analysis, performed by nuclear magnetic resonance spectroscopy $\left({ }^{1} \mathrm{H}-\mathrm{NMR}\right)$, provided information concerning the metabolic variability of the samples.

\section{Results}

Table 1 shows the average characteristics of the enrolled mothers, grouped according to the fetal growth standards. Individual variables of each subject are reported in the Supplementary Materials (Table S1). No significant difference in age, body mass index (BMI), and gestational age (GA) were observed among groups.

Table 1. Characteristics of the study population, according to fetal growth standards.

\begin{tabular}{cccc}
\hline & AGA $(\boldsymbol{n}=\mathbf{4 6})$ & SGA $(\boldsymbol{n}=\mathbf{1 0})$ & LGA $(\boldsymbol{n}=\mathbf{2})$ \\
\hline Gestational age (weeks, mean $\pm \mathrm{SD})$ & $39.3 \pm 1.5$ & $39.2 \pm 1.7$ & $38.6 \pm 0.1$ \\
Maternal age $(\mathrm{y}$, mean $\pm \mathrm{SD})$ & $31.0 \pm 5.4$ & $31.4 \pm 4.9$ & $34.5 \pm 3.5$ \\
Maternal BMI $\left(\mathrm{kg} / \mathrm{m}^{2}\right.$, mean $\left.\pm \mathrm{SD}\right)$ & $24.4 \pm 4.8$ & $22.7 \pm 4.3$ & $20.2 \pm 1.7$ \\
Cesarean delivery $(\%)$ & 52 & 80 & 50 \\
Gender $(\mathrm{M} / \mathrm{F})$ & $25 / 21$ & $3 / 7$ & $2 / 0$ \\
\hline AGA: Adequate for gestational age; SGA: small for gestational age; LGA: large for gestational age
\end{tabular}

In total, 58 HBM samples were analyzed by ${ }^{1} \mathrm{H}-\mathrm{NMR}$ spectroscopy. A representative spectrum is shown in Figure 1. In order to discover intrinsic clusters and outliers within the data set, the NMR spectral data was analyzed with the unsupervised principal components analysis (PCA) approach. The 3D PCA scores plot explained $68.1 \%$ of the variation in the dataset (Figure 2). As can be seen, no clear separation of samples was observed in terms of neonatal customized centiles (Figure 2A), gender (Figure 2B), and mode of delivery (Figure 2C). For a further study of possible differences, with regard to the above-mentioned characteristics, supervised statistical methods were also applied, without achieving significant results (data not shown). 


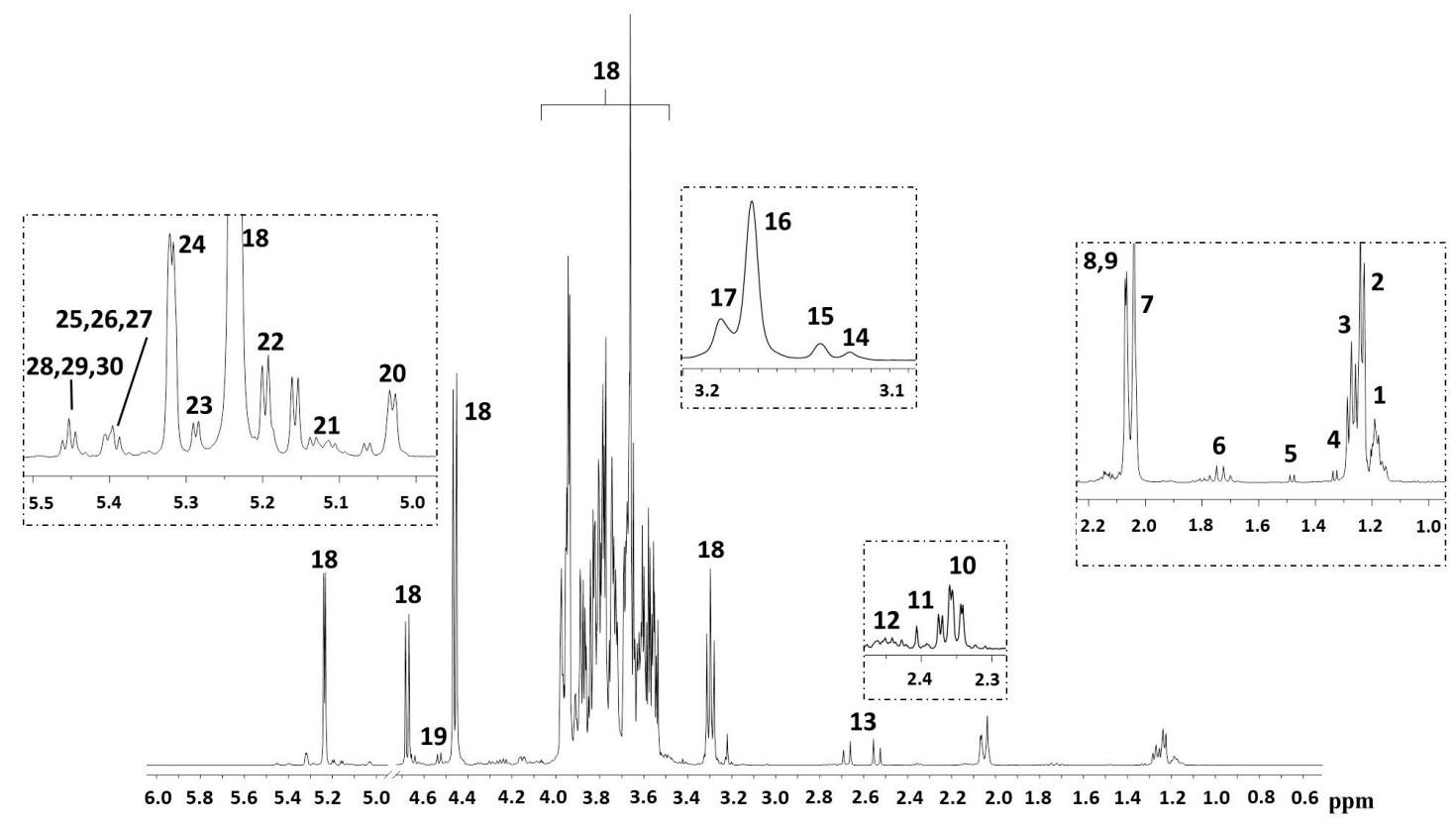

Figure 1. Representative nuclear magnetic resonance spectroscopy $\left({ }^{1} \mathrm{H}-\mathrm{NMR}\right)$ spectra of human breast milk. Chemical shift attribution was performed according to the literature $[5,6,9,10]$. Keys: (1) H-6 Fuc( $\alpha 1-3)$ Glc in $3^{\prime}$ fucosyllactose, lacto- $N$-fucopentaose V, and lacto- $N$-difucohesaose II; H-6 Fuc $(\alpha 1-3)$ GlcNAc in lacto-N-difucohesaose II; (2) H-6 Fuc $(\alpha 1-2)$ Glc in $2^{\prime}$ fucosyllactose; H-3 Fuc $(\alpha 1-2)$ Gal in $2^{\prime}$ fucosyllactose, and in lacto- $N$-fucopentaose; (3) H-6 Fuc $(\alpha 1-2)$ Gal in lacto- $N$-difucohesaose I and in lacto- $N$-difucotetraose. (4) Threonine/lactate; (5) alanine; (6) $3^{\prime}$ sialyllactose / $6^{\prime}$ sialyllactose; 7. $N$-Acetyl moieties; (8) CH3 GlcNAc( $\left.\beta 1-6\right)$ in lacto- $N$-fucopentaose; (9) $\mathrm{CH} 3 \mathrm{GlcNAc}(\beta 1-6)$ in lacto- $N$-difucohesaose I and branched; (10) glutamate; (11) succinate; (12) glutamine; (13) citrate; (14) choline; (15) O-phosphocholine; (16) glycerophosphocholine; (17) carnitine; (18) lactose; (19) H-1 Gal( $\beta 1-4) ;(20) \mathrm{H}-1$ Fuc( $\alpha 1-4)$ GlcNAC in lacto-N-difucohesaose I-II; (21) lacto- $N$-fucopentaose III and branched; (22) H-1 Fuc $(\alpha 1-2) \mathrm{Gal}$ in lacto- $N$-difucohesaose I and branched; (23) H-1 Fuc( $\alpha 1-2)$ Gal in lacto-N-difucotetraose; (24) H-1 Fuc $(\alpha 1-2)$ Gal in 2' fucosyllactose and in lacto- $N$-fucopentaose I, as well as branched; (25) H-1 Fuc $(\alpha 1-3) \alpha \mathrm{Glc}$ in lacto- $N$-difucohesaose II; (26) H-1 Fuc $(\alpha 1-3) \alpha$ Glc in $3^{\prime}$ fucosyllactose and lacto- $N$-fucopentaose V; (27) H-1 Fuc( $\left.\alpha 1-3\right) \alpha$ Glc in lacto- $N$-difucotetraose; (28) H-1 Fuc $(\alpha 1-3) \beta$ Glc in lacto- $N$-difucohesaose II; (29) H-1 Fuc( $\alpha 1-3) \beta$ Glc in $3^{\prime}$ fucosyllactose and lacto- $N$-fucopentaose V; (30) H-1 Fuc $(\alpha 1-3) \beta$ Glc in lacto- $N$-difucotetraose.

Nevertheless, exploring the three-dimensional (3D) PCA score distribution by hierarchical cluster analysis (HCA) demonstrated the presence of two main clusters, Group 1 and Group 2, containing 14 and 44 HBM samples, respectively (Figure S1 in Supplementary Materials). A subtle metabolic difference between the two groups was characterized by orthogonal projection to latent structures discriminant analysis (OPLS-DA), assigning the two clusters into classes. This allowed the construction of a very good model, which explained $85.7 \%$ and predicted $80.9 \%$ of the variation in Y (sample types), and explained 51.7\% of the variation in X (NMR response variables). Figure 3 shows the corresponding scores and coefficient loading plots. The analysis of the loadings plot indicates that the most significant contribution of the variation between the two classes arose from human milk oligosaccharides (HMOs) (Figure 3B,C): The resonances arising from $\alpha 1,3 / 4$-linked fucosyloligosaccharide units, with fucose linked only by $\alpha 1,3$ or $\alpha 1,4$ glycosidic bonds, were strongly correlated with Group 1, while those arising from $\alpha 1,2$ fucosyloligosaccharide units were strongly correlated with Group 2. In particular, the visual inspection of the corresponding NMR spectra showed a lack of signals from $\alpha 1,2$ fucosyloligosaccharides in the milk of Group 1, and the presence of peaks from all fucosylated oligosaccharides in milk of Group 2. Based on the literature data [11], these differences are indicative of secretor status in lactating mothers. Thus, Group 1 was recognized 
to be composed of milk produced from non-secretor mothers, while Group 2 included milk samples from mothers with secretory phenotype. The OPLS-DA model was validated using the response of the permutation test (Figure S2 in Supplementary Materials), and additionally by an external validation approach, splitting the data set into calibration and test sets. Table 2 shows that the misclassification analysis was able to correctly classify all samples in the test set.
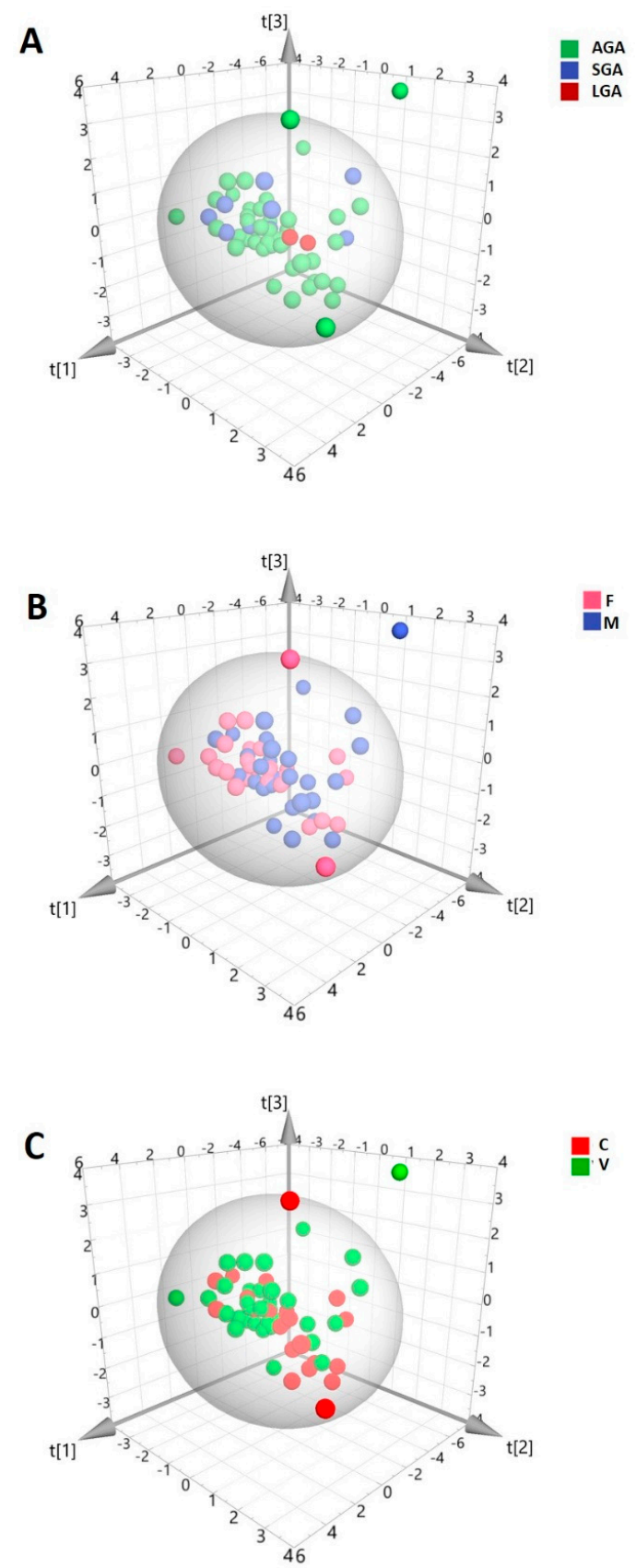

Figure 2. Three-dimensional (3D) principal components analysis (PCA) score plot of the overall data set of human breast milk (HBM) spectra from ${ }^{1} \mathrm{H}-\mathrm{NMR}$. (PC1 = 37.8\%; PC2 = 17.2\%; PC3 = 13.1\%). The scores are colored according to (A) neonatal customized centiles (AGA: appropriate for gestational age; LGA: large for gestational age; SGA: small for gestational age); (B) gender (F: female; M: male); (C) mode of delivery (C: cesarian; V: vaginal). 

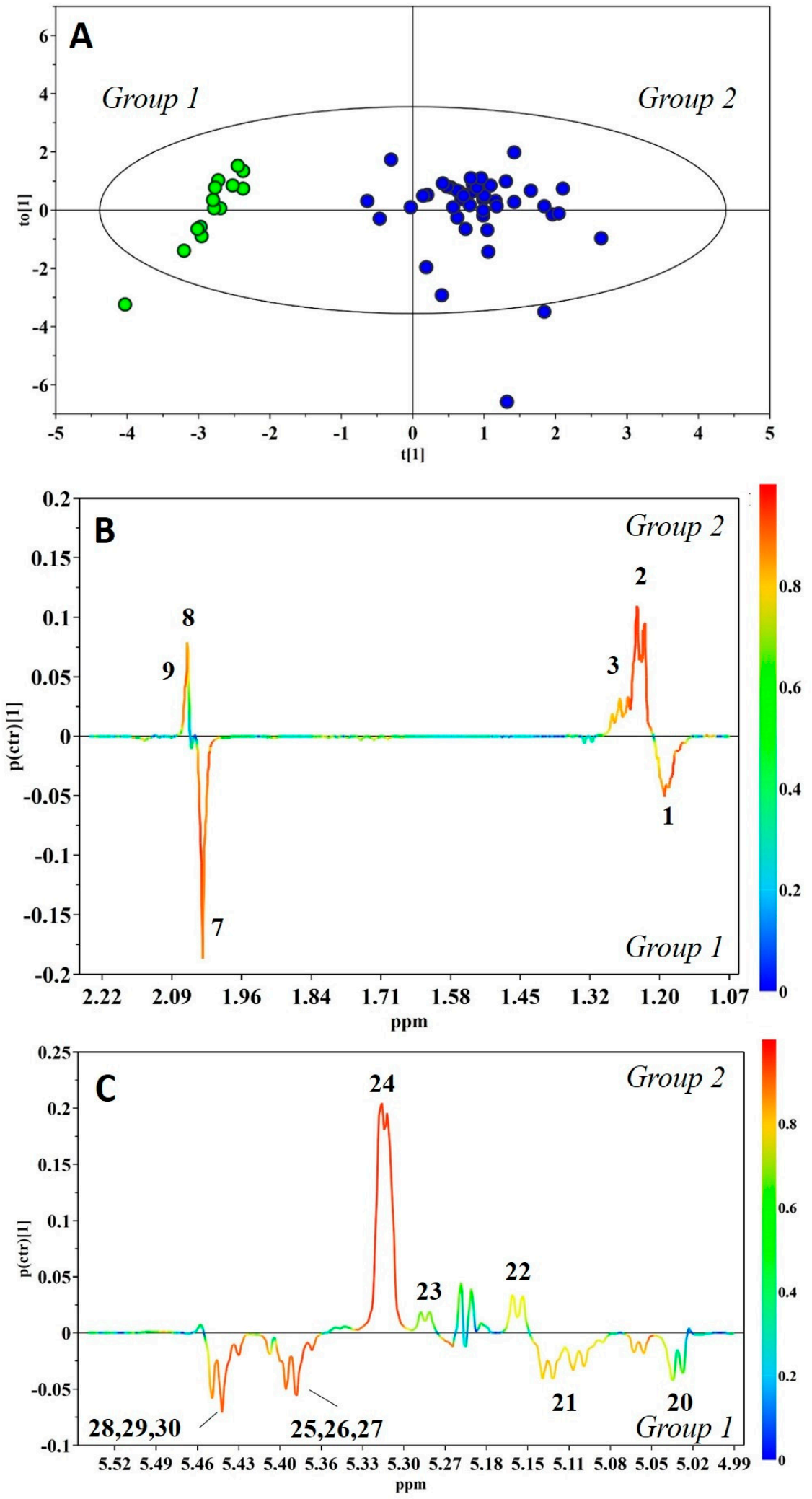

Figure 3. Orthogonal projection to latent structures discriminant analysis (OPLS-DA) score plot (A) and expansions of the loading plot of the human milk oligosaccharides' (HMOs') nuclear magnetic resonance (NMR) spectral regions (B-C) for the pair-wise comparison between Group 1 (green) and Group 2 (blue) $\left(R^{2} Y=0.857, Q^{2} Y=0.809, p<0.001\right)$. Peak codes are the same as Figure 1. 
Table 2. Average misclassification table for validation of the OPLS-DA model.

\begin{tabular}{cccc}
\hline Class & Members & Predicted & Corrected Predictions (\%) \\
\hline Group 1 & 5 & 5 & 100 \\
Group 2 & 10 & 10 & 100 \\
\hline
\end{tabular}

\section{Discussion}

HMOs are associated with the same genes that determine Lewis blood type and secretor status. Fucosyltransferase 2 (FUT2) catalyses the addition of Fuc via $\alpha 1-2$ linkages on Lewis blood group epitopes, as well as on HMOs. FUT2 is actively expressed in over $70 \%$ of the population (secretors). Milk of secretor women contains high concentrations of $\alpha 1-2$-fucosylated HMOs. One of the dominant HMOs in the milk of secretor women is $2^{\prime}$-fucosyllactose (2'FL). Non-secretors, however, do not express an active FUT2, and the milk of non-secretor women lacks $\alpha 1-2$-fucosylated HMOs. In addition, $2^{\prime} \mathrm{FL}$ is almost completely absent. Fucosyltransferase 3 (FUT3), on the other hand, catalyses the addition of Fuc via an $\alpha 1-3 / 4$ linkage (depending on the type of the underlying HMO backbone), and FUT3 can also be inactive in parts of the population (Lewis negative) [11]. Depending on the expression of active FUT2 and FUT3 enzymes, women can be separated into four groups: (1) Lewis-positive secretors (FUT2 and FUT3 active); (2) Lewis-negative secretors (FUT2 active, FUT3 inactive); (3) Lewis-positive non-secretors (FUT2 inactive, FUT3 active); and (4) Lewis-negative non-secretors (FUT2 and FUT3 inactive).

Our data indicates a higher prevalence of the secretory phenotype in the study population under investigation, in accordance with Praticò et al. [6], Smilowitz et al. [7], and Spevacek et al. [8]. This finding is consistent with global incidence; in fact, according to epidemiological studies in France, Italy, Sweden, Germany, Mexico, and Japan, $80 \%$ of women display a secretory phenotype, while in Africa, Middle East, and Bangladesh, the percentage decreases to 50\% [12]. However, the prevalence of the Lewis gene is $50 \%$ of the general population.

The secretory phenotype can represent a tool to select genetic traits that better answer the environmental factors in the population. Indeed, maternal milk with the secretory phenotype is related to an enhanced protection against some pathogens, such as E. Coli and Campylobacter. This phenomenon can explain the prevalence of the secretory phenotype in specific regions where the pathogens are endemic. For instance, the low incidence of the non-secretory phenotype in the indigenous population of Mexico could be responsible for the higher vulnerability of neonates that receive milk containing few oligosaccharides that are $\alpha 1,2$-conjugated [13].

The set of discriminant metabolites that differentiate the phenotypes of milk oligosaccharides are $2^{\prime}$ fucosyl-lactose $\left(2^{\prime} \mathrm{FL}\right)$, lactodifucotetraose (LDFT), lacto- $N$-fucopentaose I (LNFP I), and lacto- $N$-difucoesaose I (LNFDH I), which reflect the maternal secretory state and are codified by the FUT2 gene. Conversely, $3^{\prime}$ fucosyl-lactose $\left(3^{\prime} \mathrm{FL}\right)$ and lacto- $N$-difucoesaose III (LNFP III) are codified by the FUT3 gene, and they are present in the non-secretory phenotypes that are Lewis-positive.

As already mentioned, according to literature, HMOs exert several positive effects on neonates' health status. Indeed, lactofucoditetraose has anti-inflammatory properties, and suppresses platelet-induced inflammatory processes [14], while the lacto- $N$-fucopentaose is associated with decreased infant morbidity and improved growth rates [15]. In addition, $3^{\prime}$ fucosylactose seems to exert some protective effects against necrotizing enterocolitis (NEC), due to its preservation of vascular reactivity [16].

When it comes to the bacterial consumption of this particular type of saccharide, Bifidobacterium longum is one of the major consumers. This bacterial strain seems to be involved in improved response to some vaccines, and has some anti-inflammatory effects on the premature intestine. Thus, there is an indirect modulation of the immune system of the neonate host. Furthermore, it decreases the intestinal permeability, enhancing the protective mechanisms against NEC [17].

The characterization of the milk metabolome performed in the present study highlights a correlation between the phenotype expressed by the mother and the presence of specific oligosaccharides. However, no significant differences among samples were observed in terms of 
mode of delivery, gestational age, gender, and centile of the neonate. In line with the studies by Praticò et al. [6] and Smilowitz et al. [7], which show the presence of fucosylated oligosaccharides in position $\alpha 1,2$ (2'-FL, LDFT, LNFPI) in the secretory phenotype and oligosaccharides $3^{\prime}$-FL and LNFD II in the non-secretory phenotype, this study demonstrates that the milk samples of the non-secretory phenotype present $3^{\prime}$-fucosyl-lactose and other fucosylated oligosaccharides in the $\alpha 1-3 / 4$ position. In addition, the milk samples of the secretory phenotype present fucosylated oligosaccharides in the $\alpha 1-2$ position. In accordance with the study by Spevacek et al. [8], which showed the presence of fucosilated oligosaccharides, such as $3^{\prime} \mathrm{FL}, 2^{\prime} \mathrm{FL}$, and LNFP III, our study also demonstrates the above-mentioned milk oligosaccharides; however, the variation in the first month of lactation was not documented.

In the future, it would be advisable to widen the range of cases and analyze the breast milk of preterm neonates in detail through metabolomics, since from the study performed so far it has been shown that being preterm presents a decrease in the fucosylation of HMOs. Preterm neonates are a population at risk, because of the immaturity of the immune system and other tissues, which exposes them to multiple infections-NEC in particular. Neonates fed with formula milk—or if their microbiota is dominated by Proteobacteria [18], or if the maternal milk does not contain fucosylated oligosaccharides-are at a higher risk of developing this pathology. Through the identification of preterm neonates of non-secretory mothers, a specific nutrition could be introduced to get the benefits of the $\alpha 1-2$ fucosylated oligosaccharides, and to establish a more strict infectious monitoring [19].

\section{Material and Methods}

\subsection{Study Population}

This study was approved by the Alexandra Hospital ethics committee in Athens. In total, 58 women admitted to the maternity ward of the Alexandra Hospital were recruited and divided into three groups, according to the fetal growth standard. By applying GROW (Gestation Related Optimal Weight computer generated program), we calculated the customized centiles for each newborn [20]. Thus, 48 newborns were appropriate for gestational age (AGA), 2 were large for gestational age (LGA), and 10 were small for gestational age (SGA). The characteristic of the study population are reported in Table 1. Breast milk (colostrum, 5-6 mL) was collected by nursing mothers, being in good general health and nutritional state on the third or fourth day postpartum, when milk production was sufficient. Breast milk was expressed by an electric breast pump. All samples were collected during the morning following breastfeeding. Samples were transferred within $20 \mathrm{~min}$ of sampling in the laboratory into special containers on ice. Centrifugation $(20 \mathrm{~min}$ at $1500 \times \mathrm{g})$ in a refrigerated centrifuge $\left(4^{\circ} \mathrm{C}\right)$ took place immediately upon arrival. The milk's undernatant layer was separated into two equal aliquots, which were immediately stored in a deep freezer $\left(-80^{\circ} \mathrm{C}\right)$ until metabolomic analysis.

\subsection{Milk Sample Preparation}

In order to remove residual lipids and proteins, $500 \mu \mathrm{L}$ of milk samples were centrifuged at $10,000 \times g$ for $30 \mathrm{~min}$ at $4{ }^{\circ} \mathrm{C}$, using Amicon Ultra $0.5 \mathrm{~mL} 10 \mathrm{kDa}$ spin filters (Millipore, Billerica, MA, United States). Prior to filtration, filters were extensively washed with distilled water ( $500 \mu \mathrm{L}$ per filter) to deprive the membrane of the embedded glycerol. The procedure was carried out iteratively until control by NMR spectroscopy of the wash water showed no residual presence of glycerol. Each filtered sample $(350 \mu \mathrm{L})$ was mixed with $350 \mu \mathrm{L}$ of $0.1 \mathrm{M}$ phosphate buffer solution ( $\mathrm{pH} 7.4)$ containing sodium 3-trimethylsilyl-(2,2,3,3-2H4)-1-propionate (TSP) (final concentration $2 \mathrm{mM}$ ), and then $650 \mu \mathrm{L}$ were transferred into a $5 \mathrm{~mm}$ wide NMR tube.

\subsection{Data Acquisition}

The ${ }^{1} \mathrm{H}$ NMR experiments were performed at $300 \mathrm{~K}$ on a Varian UNITY INOVA 500 spectrometer (Agilent Technologies, Inc., Santa Clara, CA, United States), operating at a frequency of $499.83 \mathrm{MHz}$. 
One-dimensional (1D) ${ }^{1} \mathrm{H}$ NMR spectra were recorded using a standard pulse sequence (1D nuclear Overhauser effect spectroscopy), with pre-saturation during relaxation and mixing time for water suppression. Typically, 256 transients were acquired over a spectral width of $6000 \mathrm{~Hz}$, with a total acquisition time of $1.5 \mathrm{~s}$ and a mixing time of $0.1 \mathrm{~s}$. Prior to Fourier transformation, an exponential line-broadening factor of $0.3 \mathrm{~Hz}$ was applied to the free induction decays (FIDs). Then, spectra were phased and baseline-corrected, and the chemical shift scale was set by assigning a value of $\delta=0.00 \mathrm{ppm}$ to the signal for the internal standard TSP.

\subsection{Nuclear Magnetic Resonance Data Preparation}

The NMR milk spectra were processed using MestReNova, version 8.1 (Mestrelab Research SL, Santiago de Compostela, Spain), and misalignments in the chemical shift mainly due to pH-dependent signals were corrected. Each spectrum was integrated (binned) using $0.001 \mathrm{ppm}$ integral regions between 9.5 and $0.5 \mathrm{ppm}$, excluding the portions with the residual water $(\delta 4.6-5.2)$. Bins were normalized to the sum of the total spectral area, to compensate for the overall concentration differences. The final data set was reduced to ASCII files and converted into an Excel file.

\subsection{Multivariate Analysis of Milk Metabolic Profiles}

Data were imported into SIMCA 14 (MKS Data Analytics Solutions, Umea, Sweden) for statistical analysis. Principal component analysis (PCA) was performed to detect possible clusters or outliers. Hierarchical clustering analysis (HCA) was employed to explore the presence of similarities and dissimilarities among samples, by calculating and comparing the distances between pairs of scores in the 3D PCA space. Orthogonal projection to latent structures discriminant analysis (OPLS-DA) was applied to highlight significant biomarkers. The model quality was evaluated by (1) the goodness of fit, based on the proportion of variance in the $X\left(R^{2} X\right)$ and $Y\left(R^{2} Y\right)$ matrixes explained by the model; and (2) the goodness of prediction, based on the proportion of variance in the data that can be predicted by the model $\left(Q^{2}\right)$. The models were tested for overfitting by using $y$-table permutation testing (with 999 permutations) and a cross-validation tool [21] (the dataset was split into random subsets of training data and test data, used to build and validate the model, respectively). The significance of the models was further assed by an ANOVA based on the cross-validated predictive residuals (CV-ANOVA), with a significance threshold set to 0.05 .

\section{Conclusions}

The application of metabolomics to maternal milk is an emerging field that explores the relationship between metabolome and maternal diet, lifestyles, pathologies, and phenotype. Even if it is still in a developing stage, published studies show how metabolomics can be the key to improving knowledge about maternal milk complexities.

The aim of this study was to compare the metabolome of HBM from mothers of AGA, LGA, and SGA neonates, collected on the third or fourth day postpartum. Our results did not point out any significant difference among the milk of the three groups of mothers when analyzed in terms of fetal growth (i.e., normal, too small, too large). Conversely, a significant separation of samples into two groups, independently of the classification of newborns by weight and gestational age, was observed based on the content of HMOs (secretory versus non-secretory phenotype). The important functions of the oligosaccharides are still being investigated, and thanks to the new analytical methodologies, it will be possible to know their role in metabolic pathways in humans.

The future of research concerning maternal milk seems to include metabolomics, microbiomics, and multipotent stem cells [22,23]. From the integration of these three fields, it probably will be possible to make further progress in the understanding of the composition and functions of maternal milk, which is of major importance for the health of the newborn. If it will be possible to know which types of human oligosaccharides are produced by a mother, the management of the breastfed neonate could change forever [24]. Indeed, if some oligosaccharides are missing, with the new synthesis 
technologies they could be integrated during feeding. Furthermore, thanks to this information, it could be possible to modulate infant microbiota, from a protective point of view-especially in preterm neonates, in which the overall immaturity of the organs make them more prone to infections and other diseases, such as NEC and bronchopulmonary dysplasia [25]. In the next five years, this class of nutrient could be the main actor of the tailored nutrition and care of the newborn [26].

Supplementary Materials: The following are available online at http:/ / www.mdpi.com/2218-1989/8/4/79/s1, Figure S1: Dendogram of the hierarchical clustering analysis (HCA) performed on the 3D PCA model, Figure S2: Permutation test with 999 permutations of OPLS-DA model: $R^{2} Y$ intercept $=0.113 ; Q^{2} Y$ intercept $=-0.348$, Table S1: Characteristics of study population.

Author Contributions: Conceptualization, V.F. and A.M.-P.; Formal analysis, S.C. and F.C.M.; Investigation, S.C., S.G. (Stavroula Gavrili) and S.G. (Sofia Georgantzi); Project administration, A.D., D.B. and A.M.-P.; Resources, D.B. and V.F.; Supervision, V.F. and A.M.-P.; Writing-original draft, A.D., D.B., R.P. and A.M.-P.; Writing-review \& editing, F.C.M. and S.C.

Funding: This research received no external funding.

Conflicts of Interest: The authors declare no conflict of interest.

\section{References}

1. Jenness, R. The composition of human milk. Semin. Perinatol. 1979, 3, 225-239. [PubMed]

2. Hassiotou, F.; Hartmann, P.E. At the Dawn of a New Discovery: The Potential of Breast Milk Stem Cells. Adv. Nutr. 2014, 5, 770-778. [CrossRef] [PubMed]

3. Thurl, S.; Munzert, M.; Henker, J.; Boehm, G.; Müller-Werner, B.; Jelinek, J.; Stahl, B. Variation of human milk oligosaccharides in relation to milk groups and lactational periods. Br. J. Nutr. 2010, 104, 1261-1271. [CrossRef] [PubMed]

4. Le Doare, K.; Holder, B.; Bassett, A.; Pannaraj, P.S. Mother's Milk: A Purposeful Contribution to the Development of the Infant Microbiota and Immunity. Front. Immunol 2018, 9, 361. [CrossRef] [PubMed]

5. Cesare Marincola, F.; Dessì, A.; Corbu, S.; Reali, A.; Fanos, V. Clinical impact of human breast milk metabolomics. Clin. Chim. Acta 2015, 451, 103-106. [CrossRef] [PubMed]

6. Praticò, G.; Capuani, G.; Tommassini, A.; Baldassarre, M.E.; Delfini, M.; Miccheli, A. Exploring human breast milk composition by NMR-based metabolomics. Nat. Prod. Res. 2014, 28, 95-101. [CrossRef] [PubMed]

7. Smilowitz, J.T.; O'Sullivan, A.; Barile, D.; German, J.B.; Lönnerdal, B.; Slupski, C.M. The human milk metabolome reveals diverse oligosaccharides profiles. J. Nutr. 2013, 143, 1709-1718. [CrossRef] [PubMed]

8. Spevacek, A.R.; Smilowitz, J.T.; Chin, E.L.; Underwood, M.A.; German, J.B.; Slupsky, C.M. Infant Maturity at Birth Reveals Minor Differences in the Maternal Milk Metabolome in the First Month of Lactation. J. Nutr. 2015, 145, 1698-1708. [CrossRef] [PubMed]

9. van Leeuwen, S.S.; Schoemaker, R.J.W.; Gerwig, G.J.; van Leusen-van Kan, E.J.M.; Dijkhuizen, L.; Kamerling, J.P. Rapid milk group classification by ${ }^{1} \mathrm{H}$ NMR analysis of Le and $\mathrm{H}$ epitopes in human milk oligosaccharide donor samples. Glicobyology 2014, 24, 728-739. [CrossRef] [PubMed]

10. Wu, J.; Domellöf, M.; Zivkovic, A.M.; Larsson, G.; Öhman, A.; Nording, M.L. NMR-based metabolite profiling of human milk: A pilot study of methods for investigating compositional changes during lactation. Biochem. Biophys. Res. Commun. 2016, 469, 626-632. [CrossRef] [PubMed]

11. Castaniz-Muñoz, E.; Martin, M.J.; Prieto, P.A. 2-fucosillactose: An abundant, genetically determined soluble glycan present in human milk. Nutr. Rev. 2013, 71, 773-789. [CrossRef] [PubMed]

12. Kunz, C.; Rudloff, S.; Baier, W.; Klein, N.; Strobel, S. Oligosaccharides in human milk: Structural, functional, and metabolic aspects. Annu. Rev. Nutr. 2000, 20, 699-722. [CrossRef] [PubMed]

13. Morrow, A.L.; Meinzen-Derr, J.; Huang, P.; Schibler, K.R.; Cahill, T.; Keddache, M.; Kallapur, S.G.; Newburg, D.S.; Tabangin, M.; Warner, B.B.; et al. Fucosyltransferase 2 non-secretor and low secretor status predicts severe outcomes in premature infants. J. Pediatr. 2011, 158, 745-751. [CrossRef] [PubMed]

14. Newburg, D.S.; Tanritanir, A.C.; Chakrabarti, S. Lactodifucotetraose, a human milk oligosaccharide, attenuates platelet function and inflammatory cytokine release. J. Thromb. Thrombolysis. 2016, 42, 46-55. [CrossRef] [PubMed] 
15. Davis, J.C.; Lewis, Z.T.; Krishnan, S.; Bernstein, R.M.; Moore, S.E.; Prentice, A.M.; Mills, D.A.; Lebrilla, C.B.; Zivkovic, A.M. Growth and Morbidity of Gambian Infants are Influenced by Maternal Milk Oligosaccharides and Infant Gut Microbiota. Sci. Rep. 2017, 7, 40466. [CrossRef] [PubMed]

16. Vandaraj, S.; Helal, M.; Duska-McEwen, G.O.; Boslett, J.; Pereira, S.L.; Buck, R.H.; Ahmed, N.; Zweier, J.L. The Human Milk Oligosaccharide 3-fucosillactose facilitates pereservation of Nitric Oxide-Induced Vasodilation in Aortic Vessels in Vitro. FASEB J. 2017, 31, 1.

17. Sela, D.A. Bifidobacterial utilization of human milk oligosaccharides. Int. J. Food Microbiol. 2011, 149, 58-64. [CrossRef] [PubMed]

18. Quigley, M.A.; Henderson, G.; Anthony, M.Y.; McGuire, W. Formula milk versus donor breast milk for feeding preterm or low birth weight infants. Cochrane. Database Syst. Rev. 2007, 17, CD002971.

19. De Leoz, M.L.A.; Gaerlan, S.C.; Strum, J.S.; Dimapasoc, L.M.; Mirmiran, M.; Tancredi, D.J.; Smilowitz, J.T.; Kalanetra, K.M.; Mills, D.A.; German, J.B.; et al. Lacto-N-Tetraose, Fucosylation, and Secretor Status are Highly Variable in Human Milk Oligosaccharides from Women Delivering Preterm. J. Prot. Res. 2012, 11, 4662-4672. [CrossRef] [PubMed]

20. Gardosi, J.; Mongelli, M.; Wilcox, M.; Chang, A. An adjustable fetal weight standard. Ultrasound. Obstet. Gynecol. 1995, 6, 168-174. [CrossRef] [PubMed]

21. Westerhuis, J.A.; Hoefsloot, H.C.J.; Smit, S.; Vis, D.J.; Smilde, A.K.; van Velzen, E.J.J.; van Duijnhoven, J.P.M.; van Dorsten, F.A. Assessment of PLSDA cross validation. Metabolomics 2008, 4, 81-89. [CrossRef]

22. Fanos, V.; Pintus, R.; Reali, A.; Dessì, A. Miracles and Mysteries of Breastmilk from Egyptian to 3M's (Metabolomics, Microbiomics, Multipotent Stem Cells). J. Pediatr. Neonat. Med. 2017, 6, e0606204.

23. Bardanzellu, F.; Fanos, V.; Reali, A. "Omics” in Human Colostrum and Matue Milk: Looking to Old data with new eyes. Nutrients 2017, 9, E843.

24. Fanos, V.; Reali, A.; Marcialis, M.A.; Bardanzellu, F. What you have to know about Human Milk Oligosaccharides. J. Pediart. Neonat. Individual. Med. 2018, 7, e0707137.

25. Marincola, F.C.; Noto, A.; Caboni, P.; Reali, A.; Barberini, L.; Lussu, M.; Murgia, F.; Santoru, M.L.; Atzori, L.; Fanos, V. A metabolomic study of preterm human and formula milk by high resolution NMR and GC/MS analysis: Preliminary results. J. Matern. Fetal. Neonatal. Med. 2012, 25, 62-67. [CrossRef] [PubMed]

26. Bardanzellu, F.; Fanos, V.; Stringini, F.A.L.; Artini, P.G.; Peroni, D.G. Human Breast Milk: Exploring the Linking Ring Among Emerging Components. Front. Pediatr. 2018, 6, 215. [CrossRef] [PubMed] 